\title{
Pathways for Emotions: Specializations in the Amygdalar, Mediodorsal Thalamic, and Posterior Orbitofrontal Network
}

\author{
Clare Timbie ${ }^{1,2}$ and Helen Barbas ${ }^{1,2}$ \\ ${ }^{1}$ Department of Anatomy and Neurobiology, Boston University School of Medicine, Boston, Massachusetts 02118, and ${ }^{2}$ Neural Systems Laboratory, \\ Department of Health Sciences, Boston University, Boston, Massachusetts 02215
}

The primate amygdala projects to posterior orbitofrontal cortex (pOFC) directly and possibly indirectly through a pathway to the magnocellular mediodorsal thalamic nucleus (MDmc), which may convey signals about the significance of stimuli. However, because $\mathrm{MDmc}$ receives input from structures in addition to the amygdala and MDmc projects to areas in addition to pOFC, it is unknown whether amygdalar pathways in MDmc innervate pOFC-bound neurons. We addressed this issue using double- or triple-labeling approaches to identify pathways and key cellular and molecular features in rhesus monkeys. We found that amygdalar terminations innervated labeled neurons in MDmc that project to pOFC. Projection neurons in MDmc directed to pOFC included comparatively fewer "core" parvalbumin neurons that project focally to the middle cortical layers and more "matrix" calbindin neurons that project expansively to the upper cortical layers. In addition, a small and hitherto unknown pathway originated from MDmc calretinin neurons and projected to pOFC. Further, whereas projection neurons directed to $\mathrm{MDmc}$ and to $\mathrm{pOFC}$ were intermingled in the amygdala, none projected to both structures. Larger amygdalar neurons projected to MDmc and expressed the vesicular glutamate transporter 2 (VGLUT2), which is found in highly efficient "driver" pathways. In contrast, smaller amygdalar neurons directed to pOFC expressed VGLUT1 found in modulatory pathways. The indirect pathway from the amygdala to pOFC via MDmc may provide information about the emotional significance of events and, along with a parallel direct pathway, ensures transfer of signals to all layers of pOFC.

Key words: amygdala; emotion; orbitofrontal cortex; prefrontal cortex; primate; thalamus

\section{Significance Statement}

The amygdala - the brain's center for emotions - is strongly linked with the orbital cortex, a region associated with social interactions. This study provides evidence that a robust pathway from the amygdala reaches neurons in the thalamus that link directly with the orbital cortex, forming a tight tripartite network. The dual pathways from the amygdala to the orbital cortex and to the thalamus are distinct by morphology, neurochemistry, and function. This tightly linked network suggests the presence of fool-proof avenues for emotions to influence high-order cortical areas associated with affective reasoning. Specific nodes of this tripartite network are disrupted in psychiatric diseases, divorcing areas that integrate emotions and thoughts for decisions and flexible behavior.

\section{Introduction}

The amygdala is critical for processing the affective content of stimuli (Davis and Whalen, 2001; Baxter and Murray, 2002; Sal-

Received June 3, 2015; revised July 15, 2015; accepted July 22, 2015.

Author contributions: C.T. and H.B. designed research; C.T. and H.B. performed research; C.T. and H.B. analyzed data; C.T. and H.B. wrote the paper.

This work was supported by the National Institute of Mental Health-National Institutes of Health (National Research Service Award F30MH093002 to C.T. and Grant R01MH057414 to H.B.), the National Institute of Neurological Disorders and Stroke-National Institutes of Health (Grant R01NS024760 to H.B.), and the Center of Excellence for Learning in Education, Science and Technology (National Science Foundation Science of Learning Center Grant OMA-0835976). We thank Ron Killiany for assistance with MR imaging; Marcia Feinberg for assistance with electron microscopy; Eric Wong for technical assistance; and Basilis Zikopoulos, Maria Medalla, Jamie G. Bunce, Miguel A. Garcia-Cabezas, and Yohan John for comments on the manuscript.

The authors declare no competing financial interests.

Correspondence should be addressed to Helen Barbas, Department of Health Sciences, Boston University, 635 Commonwealth Avenue, Room 431, Boston, MA 02215. E-mail: barbas@bu.edu. zman and Fusi, 2010; West et al., 2011). The strongest amygdalar pathways reach posterior orbitofrontal cortex (pOFC), where affective signals and sensory information converge and enable representation of task state (Davis and Whalen, 2001; Ghashghaei et al., 2007; Wilson et al., 2014). The primate amygdala and pOFC are also interconnected with the medial (magnocellular) mediodorsal (MDmc) thalamic nucleus (Porrino et al., 1981; Aggleton and Mishkin, 1984; McFarland and Haber, 2002; Miyashita et al., 2007; Izquierdo and Murray, 2010), forming a tripartite network.

A key unanswered question is whether the amygdalar pathway to MDmc targets neurons that project to pOFC, which could convey information about the significance of events. This question arises because $\mathrm{MDm}$ receives input from several structures 
Table 1. Injection sites in pOFC, amygdala, and thalamus

\begin{tabular}{lllll}
\hline Case/hemisphere & Injection site/division & Tracer & Sex & Age (y) \\
\hline BC/right & A13 & FE & $\mathrm{M}$ & 3 \\
BJ/right & p0FC/orbital pro-isocortex & LY & $\mathrm{F}$ & 2 \\
BK/right & p0FC/orbital pro-isocortex & $\mathrm{CBL}$ & $\mathrm{F}$ & 2.5 \\
$\mathrm{BM} /$ left & p0FC/orbital pro-isocortex & $\mathrm{FE}$ & $\mathrm{F}$ & 3.5 \\
$\mathrm{BP} /$ right & p0FC/orbital pro-isocortex & $\mathrm{FR}$ & $\mathrm{F}$ & 3.5 \\
BL/right & Amygdala/cortical, basomedial & $\mathrm{FR}$ & $\mathrm{M}$ & 3 \\
$\mathrm{BM} /$ left & Amygdala/basolateral, lateral & $\mathrm{FR}$ & $\mathrm{F}$ & 3.5 \\
BN/right & Amygdala/basomedial, basolateral & $\mathrm{FE}$ & $\mathrm{M}$ & 2 \\
$\mathrm{BS} /$ left & p0FC/orbital pro-isocortex & $\mathrm{FB}$ & $\mathrm{F}$ & 3.5 \\
$\mathrm{BS} /$ left & Mediodorsal thalamus/magnocellular, & $\mathrm{FR}$ & $\mathrm{F}$ & 3.5 \\
& parvicellular & & & \\
\hline
\end{tabular}

and projects to areas in addition to pOFC (Mitchell and Chakraborty, 2013). Previous studies have shown that amygdalar and thalamic MD pathways in pOFC are complementary. Pathways from the amygdala terminate most densely in the upper layers of pOFC, whereas thalamic pathways terminate mostly in the middle layers (Giguere and Goldman-Rakic, 1988; McFarland and Haber, 2002; Timbie and Barbas, 2014). If projections from $\mathrm{MDmc}$ represent an indirect route for amygdalar input to pOFC, then the amygdala may have laminar-specific effects in pOFC through the direct and indirect pathways. In this context, the direct amygdalar pathway targets functionally distinct classes of inhibitory neurons in the upper and middle-deep layers of pOFC (Timbie and Barbas, 2014).

Also unexplored are functionally relevant features of the tripartite circuit that links the amygdala with MDmc and both structures with pOFC. One of these pertains to dual excitatory pathway systems in the thalamus that project to the cortex. The classical thalamocortical pathway in sensory systems originates in excitatory PV neurons and projects to the middle cortical layers. A parallel pathway from excitatory CB neurons projects to the upper cortical layers (Jones, 1998). The relative prevalence of PV and CB neurons that give rise to dual projection systems from the high-order MDmc thalamic nucleus to pOFC is unknown.

Another issue pertains to the neurochemical features of the dual amygdalar pathways to MDmc and to pOFC. Available data hint that the two pathways differ in rats (McDonald, 1987) and primates (Miyashita et al., 2007); however, there is no information about the identity of these pathways with respect to recently described functionally distinct classes of glutamatergic pathways (Fremeau et al., 2004; Santos et al., 2009).

To investigate these key features of the tripartite circuit, we addressed the following questions: (1) is there evidence of a sequential amygdalar-MDmc-pOFC pathway? (2) what is the relative prevalence of $\mathrm{PV}$ and $\mathrm{CB}$ projection neurons in MD directed to pOFC? and (3) do the dual amygdalar pathways to pOFC and to $\mathrm{MD}$ arise from the same or different neuronal populations? We provide evidence of salient differences in these pathways that are associated with affective reasoning for flexible behavior and are often disrupted in psychiatric diseases.

\section{Materials and Methods}

Surgery, tracer injections, and tissue processing

Experiments were conducted on eight rhesus monkeys (Macaca mulatta) of both sexes aged 2-3.5 years, which were injected with 1-2 distinct neural tracers (see Fig. 1, Table 1). Experiments were conducted according to the Guide for the Care and Use of Laboratory Animals (National Research Council, 2011). Experimental methods were approved by the Institutional Animal Care and Use Committee at Boston University
School of Medicine, Harvard Medical School, and New England Primate Research Center. Procedures involving animals were designed to reduce the number of animals needed and to minimize animal suffering.

Before surgery, we obtained MRIs after animal sedation with ketamine hydrochloride (10-15 mg/kg, i.m.), propofol anesthesia (loading dose $2.5-5 \mathrm{mg} / \mathrm{kg}$, i.v.; infusion rate, $0.25-0.4 \mathrm{mg} / \mathrm{kg} / \mathrm{min}$ ) and placement in a stereotaxic apparatus (1430M; David Kopf Instruments). The images were used to calculate stereotaxic coordinates for the injection sites. Surgery for injection of tracers was conducted under sterile conditions and animals were monitored continuously for respiratory rate, oxygen saturation, heart rate, and temperature. The animals were sedated with ketamine hydrochloride, anesthetized with isofluorane, and positioned in the same stereotaxic apparatus as for imaging (the Kopf 1430M). A small opening was made in the skull and dura to inject neural tracers into amygdalar nuclei, pOFC, or MD using Hamilton syringes (10 $\mu \mathrm{l})$. Tracers included: $3 \%$ dilutions of fast blue (FB, $2 \mu \mathrm{l}$; Polysciences) or $10 \%$ dilutions of lucifer yellow dextran (LY, $4 \mu \mathrm{l}, 10 \mathrm{kDa}$; Invitrogen), fluoroemerald (FE, fluorescein dextran, $3 \mu \mathrm{l}$ of $10 \mathrm{kDa}$ or a mixture of 3 and $10 \mathrm{kDa}$; Invitrogen), fluororuby (FR, tetramethylrhodamine dextran, 3-4 $\mu \mathrm{l}$ of a mixture of 3 and $10 \mathrm{kDa}$; Invitrogen), or cascade blue dextran (CBL, $6 \mu \mathrm{l}$ of a mixture of 3 and $10 \mathrm{kDa}$; Invitrogen). We adjusted the volume of dye injected to account for differences in diffusion pattern among the dyes. Injection sites were small relative to the size of the target area (Fig. 1). Hamilton syringes were filled with dye and then a small amount of air to prevent transfer of dye to dorsal structures during injection. Syringes were left in place for 5-10 min after injection to allow absorption locally and to prevent backward suction of the dye in the needle after injection.

After $18 \mathrm{~d}$, the animals were anesthetized with sodium pentobarbital and perfused transcardially with $4 \%$ paraformaldehyde, $0.2 \%$ glutaraldehyde in $0.1 \mathrm{~m} \mathrm{PBS}, \mathrm{pH}$ 7.4. The brain was removed, cryoprotected in ascending sucrose solutions ( $10-30 \%$ sucrose $\mathrm{w} / \mathrm{v}$ in $0.1 \mathrm{M} \mathrm{PBS,} \mathrm{pH} \mathrm{7.4,}$ with $0.05 \%$ sodium azide; Sigma-Aldrich; Rosene et al., 1986), frozen in isopentane (Fisher Scientific) at $-80^{\circ} \mathrm{C}$, and cut on a freezing microtome (AO Scientific Instruments/Reichert Technologies) in $50 \mu \mathrm{m}$ coronal sections forming 10 matched series. Sections were stored free floating at $-20^{\circ} \mathrm{C}$ in a solution of $30 \%$ ethylene glycol, $30 \%$ glycerol, $0.05 \%$ sodium azide in $0.05 \mathrm{M}$ phosphate buffer, $\mathrm{pH} 7.4$.

\section{Bright-field and confocal microscopy}

To identify overlap between amygdalar axons and neurons in MDmc that project to $\mathrm{POFC}$, the thalamus was removed after perfusion and before cryoprotection, postfixed for $3 \mathrm{~d}$ in $4 \%$ paraformaldehyde, embedded in $7 \%$ agarose (Electron Microscopy Sciences), and cut on a vibratome at $100 \mu \mathrm{m}$ (case BM-L). Sections were mounted on glass slides and coverslipped while damp with Prolong Gold Antifade mounting medium (Invitrogen). Areas of overlap between amygdalar axons and neurons in MDmc that project to pOFC were imaged using laser scanning confocal microscopy (Fluoview FV-300, Olympus; LSM-510, Carl Zeiss) at a $630 \times$ to $1000 \times$ magnification. Stacks of optical sections $0.3 \mu \mathrm{m}$ thick were acquired in MDmc to identify appositions between amygdalar axon terminals and neurons in MDmc that project to pOFC. Appositions were defined as close contacts that were visible at all angles between a labeled bouton and labeled dendrite. FE tracer was visualized with a $488 \mathrm{~nm}$ argon laser and FR tracer with a $568 \mathrm{~nm}$ krypton laser (Olympus) or a 543 $\mathrm{nm}$ helium neon laser (Zeiss). We also mapped the overlap between labeled neurons in MDmc that project to pOFC and amygdalar fibers in MD. We used exhaustive sampling of one of four sections through MD and identified the proportion of neurons in MDmc that project to pOFC that were within $100 \mu \mathrm{m}$ of an amygdalar fiber (StereoInvestigator 10, MBF Biosciences; BX60, Olympus).

In the amygdala, we mapped labeled neurons directed to pOFC and to $\mathrm{MD}$ to investigate whether amygdalar neurons project to both structures (case BS-L). We used exhaustive sampling of one in 10 sections through the amygdala and a microscope equipped with a motorized stage to identify labeled neurons throughout the amygdala (StereoInvestigator 10, MBF Biosciences; BX60, Olympus). We also estimated the area of each labeled neuron using the nucleator method to identify any population differences in neuron size (StereoInvestigator 10). 
Immunohistochemistry. To label thalamic projection neurons and their content of $\mathrm{CB}, \mathrm{PV}$, or $\mathrm{CR}$, free floating $50 \mu \mathrm{m}$ sections containing $\mathrm{MD}$ were rinsed in $\mathrm{PB}$ and then incubated in $0.01 \mathrm{M}$ sodium citrate buffer, $\mathrm{pH}$ 8.5 , at $35-45^{\circ} \mathrm{C}$ for $30 \mathrm{~min}$ (Jiao et al., 1999). A $30 \mathrm{~min}$ incubation with $0.3 \%$ hydrogen peroxide in $0.01 \mathrm{~m} \mathrm{PBS}, \mathrm{pH} 7.4$, quenched endogenous peroxidases. Sections were rinsed in PBS and then incubated for $1 \mathrm{~h}$ at $4^{\circ} \mathrm{C}$ in $0.05 \mathrm{M}$ glycine (Sigma-Aldrich) and preblocked for $1 \mathrm{~h}$ at $4^{\circ} \mathrm{C}$ in $5 \%$ normal goat serum (NGS; Vector Laboratories), 5\% bovine serum albumin (BSA; Sigma-Aldrich), and 0.2\% Triton-X (Sigma-Aldrich) in $0.01 \mathrm{M}$ PBS. Tracers and calcium-binding proteins were bound overnight at $4^{\circ} \mathrm{C}$ with primary antibodies (FE, FR, CBL, or LY: $1: 800$ in $1 \%$ NGS, $1 \%$ BSA, and $0.1 \%$ Triton-X in PBS; rabbit polyclonal, Invitrogen; and CB, CR, or PV: 1:2000, mouse monoclonal, Swiss Antibodies). For each incubation, primary and secondary antibody penetration was enhanced with $8 \mathrm{~min}$ runs ( $3 \mathrm{~min}$ on, $2 \mathrm{~min}$ off, $3 \mathrm{~min}$ on) in a temperaturecontrolled variable wattage microwave oven $\left(150 \mathrm{~W}\right.$ at $4^{\circ} \mathrm{C}$; Pelco Biowave with ColdSpot and ThermoCube; Ted Pella). Next, the tissue was rinsed in PBS and incubated overnight at $4^{\circ} \mathrm{C}$ in secondary antibodies conjugated with fluorescent label (1:100 in $1 \%$ NGS, $1 \%$ BSA, and $0.1 \%$ Triton-X in PBS; Alexa Fluor 568 goat anti-rabbit or goat anti-mouse IgG and Alexa Fluor 488 goat anti-mouse or goat anti-rabbit IgG; Invitrogen). Sections were rinsed in PB, mounted on gelatin-coated glass slides, and coverslipped while damp with Prolong Gold Antifade mounting medium (Invitrogen) or FluorSave reagent (Millipore).

For analysis of vesicular glutamate transporters 1 and 2 (VGLUT1 and VGLUT2), sections were incubated in sodium citrate buffer and glycine and blocked as above and any BDA tracer was blocked using avidinbiotin blocking solution (AB blocking kit; Vector Laboratories). Sections were then incubated overnight in primary antibodies to VGLUT1 (1: 2000 in $1 \%$ NGS, $1 \%$ BSA, and $0.1 \%$ Triton-X in PBS; anti VGLUT1 rabbit polyclonal; MAb Technologies) or VGLUT2 (1:2000 in 1\% NGS, $1 \%$ BSA, and $0.1 \%$ Triton-X in PBS; anti VGLUT2 rabbit polyclonal; MAb Technologies). Sections were rinsed in PBS and then incubated for $2 \mathrm{~h}$ at $4^{\circ} \mathrm{C}$ in biotinylated secondary antibodies (1:200 in $1 \% \mathrm{NGS}, 1 \%$ BSA, and $0.1 \%$ Triton-X in PBS; biotin-SP Fab fragment goat anti-rabbit IgG; Jackson ImmunoResearch). Sections were then incubated for $1 \mathrm{~h}$ at $25^{\circ} \mathrm{C}$ with avidin-biotin horseradish peroxidase (AB-HRP, Vectastain Elite ABC kit; Vector Laboratories) at a 1:100 dilution in PBS, followed by rinses in PBS. VGLUT signal was amplified through incubation in biotinylated tyramide signal amplification solution (1:4000 in PBS, TSA Biotin Kit; PerkinElmer) with $0.005 \%$ hydrogen peroxide for $20 \mathrm{~min}$ at $25^{\circ} \mathrm{C}$. This step also ensured that any rabbit-binding sites on the primary or secondary antibody were blocked before incubation with a second rabbit primary antibody. Sections were then rinsed in PBS and incubated overnight at $4^{\circ} \mathrm{C}$ in antibodies to label tracers (FE or FR: $1: 800$ in $1 \%$ NGS, $1 \%$ BSA, and $0.1 \%$ Triton-X in PBS; rabbit polyclonal; Invitrogen). After rinses in PBS, sections were incubated overnight at $4^{\circ} \mathrm{C}$ in streptavidin conjugates to visualize VGLUT (1:100 Alexa Fluor 647 streptavidin; Invitrogen) and secondary antibodies to visualize tracers [1:100 in $1 \%$ NGS, $1 \%$ BSA, and $0.1 \%$ Triton-X in PBS; Alexa Fluor 488-AffiniPure Fab fragment goat anti-rabbit IgG (FE) or Rhodamine Red-X-AffiniPure Fab fragment goat anti-rabbit IgG; Jackson Immuno Research). In control experiments, omission of the primary antibodies and incubating in secondary antibody solutions showed no immunolabeling.

Data analysis. In four to eight evenly spaced sections, we analyzed retrogradely labeled pOFC projection neurons in $\mathrm{MD}$ also labeled for $\mathrm{CB}$ or PV (three cases: BJ-R, BK-R, BP-R) through the entire rostral-caudal extent of MD (Olszewski, 1952). We analyzed one additional case (BC-R) with an injection site confined to layers 5-6 and white matter below orbital area 13 for comparison. In one case (BJ-R), an additional series of sections was labeled for CR. For each section, all retrogradely labeled neurons in MD were counted at 200× magnification and colocalization with $\mathrm{CB}$ or PV was determined using an epifluorescence microscope and digital camera (BX51 and DP70 digital camera; Olympus).

To study colocalization between fluorescently labeled tracer and VGLUT1 or VGLUT2, we used laser scanning confocal microscopy (LSM-510; Carl Zeiss). Stacks of optical sections $0.3 \mu \mathrm{m}$ thick were acquired in patches of labeled fibers in upper layers $2-3 \mathrm{a}$ of pOFC or in $\mathrm{MDmc}$ at $630 \times$ magnification. Alexa Fluor 488 conjugates were visual- ized with a $488 \mathrm{~nm}$ argon laser, rhodamine red X conjugates with a 543 $\mathrm{nm}$ helium neon laser, and Alexa Fluor 647 conjugates with a $633 \mathrm{~nm}$ helium neon laser. Images were deconvolved to remove noise and fluorescent halo (AutoDeblur, version X1.4.1; MediaCybernetics). All labeled boutons were counted and ImageJ and ColocalizeRGB plugin were used to detect colocalization between labeled boutons and VGLUT1 or VGLUT2 (Rasband, 1997-2014). $\chi^{2}$ tests were used to compare proportions of VGLUT1 or VGLUT2 colocalized boutons in pOFC and MDmc. Student's $t$ test (two-tailed) was used to test for differences in the two populations of projection neurons in the amygdala.

\section{Electron microscopy}

Immunohistochemistry and embedding. To study pathways in the electron microscope (EM), we used triple immunohistochemistry to identify tracers with DAB (which appears as uniform dark precipitate under EM) and calcium binding proteins (CB or PV) using gold labeling with silver enhancement (forms clumps of round particles) and tetramethylbenzidine (TMB) staining (forms rod-shaped precipitate). Tissue sections were incubated as above in $0.01 \mathrm{M}$ sodium citrate, $\mathrm{pH} 8.5$ (30 min at $\left.35^{\circ} \mathrm{C}\right), 0.05 \mathrm{M}$ glycine $\left(1 \mathrm{~h}\right.$ at $\left.4^{\circ} \mathrm{C}\right)$, and $\mathrm{BDA}$ tracer was blocked with $\mathrm{AB}$ blocking solutions. Background binding was blocked with incubation for $1 \mathrm{~h}$ at $4^{\circ} \mathrm{C}$ in $5 \%$ NGS, 5\% BSA, $0.025 \%$ Triton X-100 (Roche), $0.1 \%$ acetylated BSA-c (Aurion), and 3.5\% mouse blocking reagent (MOM basic kit; Vector Laboratories) in PBS. Sections were then bound overnight at $4^{\circ} \mathrm{C}$ with antibodies for tracers (FE, FR, or LY: 1:800 in 1\% NGS, $1 \%$ BSA, $0.1 \%$ BSA-c, $0.025 \%$ Triton X-100, and $8 \%$ MOM protein concentrate, MOM basic kit; Vector Laboratories in PBS; rabbit polyclonal IgG; Invitrogen) and one of two calcium-binding proteins (CB or PV: 1:2000, mouse monoclonal IgG; Swant). All primary and secondary antibody incubations included an $8 \mathrm{~min}$ microwave run as above.

Sections were rinsed in PBS and then incubated for $6 \mathrm{~h}$ at $25^{\circ} \mathrm{C}$ with biotinylated secondary antibodies for tracers [1:200 in 1\% NGS, $1 \%$ BSA, $0.1 \%$ BSA-c, $0.025 \%$ Triton X-100, $8 \%$ MOM protein concentrate, and $0.1 \%$ coldwater fish gelatin (Aurion) in PBS; biotinylated goat antirabbit IgG (Vector Laboratories) and gold-conjugated secondary antibodies for calcium-binding proteins (1:50 UltraSmall ImmunoGold $\mathrm{F}(\mathrm{ab})$ fragment of goat anti-mouse IgG; Aurion). Sections were then postfixed with $3 \%$ glutaraldehyde and $1 \%$ paraformaldehyde in $\mathrm{PB}$ in a microwave oven $\left(2 \mathrm{~min}\right.$ at $\left.150 \mathrm{~W}, 4^{\circ} \mathrm{C}\right)$. Sections were rinsed in glycine $(5$ $\mathrm{min})$ and rinsed in $\mathrm{PB}(2 \times 10 \mathrm{~min})$, followed by enhancement conditioning solution (ECS, 1:10, $2 \times 10 \mathrm{~min}$; Aurion). Gold-conjugated proteins were visualized by silver enhancement for 60-90 min (R-Gent SE-EM; Aurion) and the tissue was then rinsed in ECS $(2 \times 10 \mathrm{~min})$ and then $\mathrm{PB}(2 \times 10 \mathrm{~min})$. Tracers were visualized with $\mathrm{DAB}$ as above. For all rinses, after silver enhancement, we used $0.1 \mathrm{M} \mathrm{PB}, \mathrm{pH} 7.4$, and, in some pieces of tissue, the order of labeling was reversed to control for any attraction between gold and biotin. Any remaining biotin-binding sites were blocked with $\mathrm{AB}$-blocking solutions and then any remaining mouse-binding sites were blocked with incubation for $1 \mathrm{~h}$ at $4^{\circ} \mathrm{C}$ in $3.5 \%$ mouse-blocking reagent (5\% NGS, 5\% BSA, $0.025 \%$ Triton X-100, and $0.1 \% \mathrm{BSA}-\mathrm{c}$ in $\mathrm{PB})$.

Sections were incubated overnight at $4^{\circ} \mathrm{C}$ with antibody for a second calcium-binding protein (CB or PV: 1:2000 in 1\% NGS, 1\% BSA, $0.1 \%$ BSA-c, $0.025 \%$ Triton X-100, and 8\% MOM protein concentrate in PB; mouse monoclonal IgG; Swant), followed by rinses in PB and incubation for $1-2 \mathrm{~h}$ at $25^{\circ} \mathrm{C}$ in biotinylated secondary antibody (1:200 in 1\% NGS, $1 \%$ BSA, $0.1 \%$ BSA-c, $0.025 \%$ Triton X-100, and $8 \%$ MOM protein concentrate in PB; biotinylated goat anti-mouse IgG; Vector Laboratories), rinsed in $\mathrm{PB}$, and incubated in $\mathrm{AB}-\mathrm{HRP}$ as above. These calciumbinding proteins were visualized with TMB staining as follows: sections were first incubated for $15 \mathrm{~min}$ in $0.005 \% \mathrm{TMB}$ (Sigma-Aldrich), $0.004 \%$ ammonium chloride (Sigma-Aldrich), and 5\% ammonium paratungstate (Sigma-Aldrich) in $0.1 \mathrm{M} \mathrm{PB}, \mathrm{pH} 6.0$, and then incubated for 1-5 min in the same solution plus $0.005 \%$ hydrogen peroxide (SigmaAldrich) until staining appeared. The staining was stabilized by incubating sections for $10 \mathrm{~min}$ in a solution of $0.05 \%$ DAB (SigmaFast DAB tablet; Sigma-Aldrich), $0.02 \%$ cobalt chloride (Sigma-Aldrich), $0.004 \%$ ammonium chloride (Sigma-Aldrich), and $0.005 \%$ hydrogen peroxide (Sigma-Aldrich) in $0.1 \mathrm{~m} \mathrm{~PB}, \mathrm{pH}$ 6.0. Finally, sections were rinsed in $\mathrm{PB}$ 

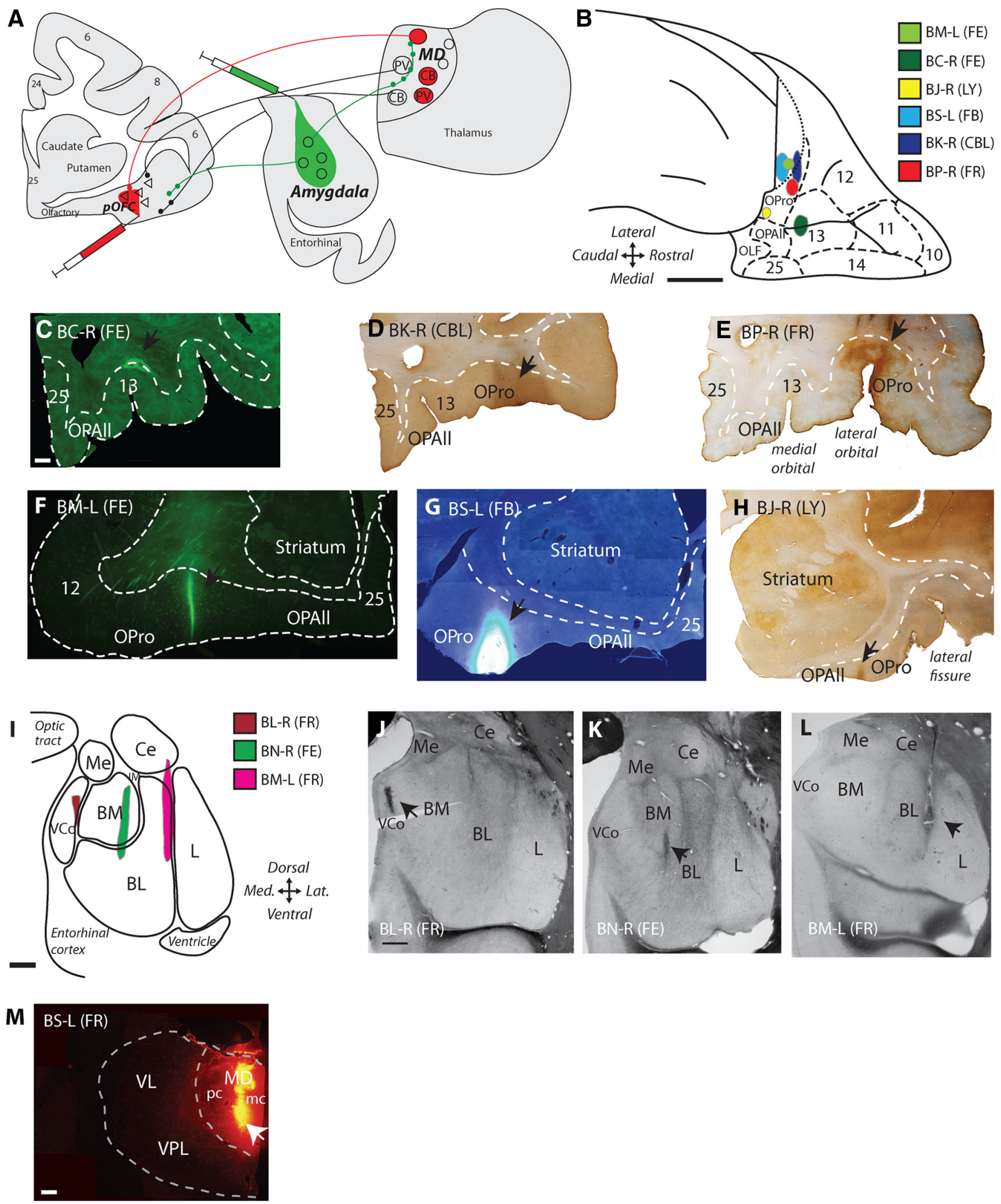

Figure 1. Label of pathways with neural tracer injections in the amygdala, $\mathrm{pOFC}$ and the thalamic $\mathrm{MD}$. $A$, Experimental design: injections of retrograde tracer in $\mathrm{p} 0 \mathrm{FC}$ and anterograde tracer in the amygdala label overlapping zones in MD. $\boldsymbol{B}$, Injection sites in pOFC shown on the orbital surface of a rhesus monkey brain. Scale bar, $5 \mathrm{~mm}$. $\boldsymbol{C}-\boldsymbol{H}$, Coronal sections through p0FC show injection sites of neural tracer in fluorescence microscopy $(\boldsymbol{C}, \boldsymbol{F}, \boldsymbol{G})$ or converted for bright-field microscopy $(\boldsymbol{D}, \boldsymbol{E}, \boldsymbol{H})$. Scale bar in $\boldsymbol{C}$ is $1 \mathrm{~mm}$ and applies to $\boldsymbol{C}-\boldsymbol{H}$. $\boldsymbol{I}$, Injection sites in the amygdala are shown schematically on a coronal section. Scale bar, $1 \mathrm{~mm}$. $\boldsymbol{J}-\boldsymbol{L}$, Fresh tissue sections in the amygdala show injection sites of neural tracers (arrows). Scale bar in $\boldsymbol{J}$ is $1 \mathrm{~mm}$ and applies to $J-\boldsymbol{L}$. $\boldsymbol{M}$, Coronal section through the thalamic MD shows injection site of FR tracer mostly in MDmc. Scale bar, $1 \mathrm{~mm}$. 
and postfixed in $6 \%$ glutaraldehyde and $2 \%$ paraformaldehyde in $\mathrm{PB}$ with a microwave oven $\left(150 \mathrm{~W}\right.$ at $\left.15^{\circ} \mathrm{C}\right)$ until the sample temperature reached $30-35^{\circ} \mathrm{C}$ (Jensen and Harris, 1989). Control experiments were conducted on tissue by omitting primary antibodies and incubating in secondary antibody solutions and no immunolabeling was found.

Sections were rinsed in $\mathrm{PB}(3 \times 20 \mathrm{~min})$ and postfixed for $15 \mathrm{~min}$ in $1 \%$ osmium tetroxide (Electron Microscopy Sciences) with $1.5 \%$ potassium ferrocyanide (Electron Microscopy Sciences) in PB with a microwave oven (100 W at $12^{\circ} \mathrm{C}, 2 \mathrm{~min}$ on, $2 \mathrm{~min}$ off, $2 \mathrm{~min}$ on) and rinsed in $\mathrm{PB}(3 \times 2 \mathrm{~min})$ and water $(3 \times 2$ $\min )$. Sections were then rinsed in $50 \%$ ethanol $(3 \times 5 \mathrm{~min})$, stained with $1 \%$ uranyl acetate $(30$ min in $70 \%$ ethanol; Electron Microscopy Sciences), and dehydrated in a series of ethanols $(90 \%, 95 \%, 100 \% ; 3 \times 5$ min each). For embedding, sections were infiltrated with propylene oxide $(2 \times 7 \mathrm{~min}$; Electron Microscopy Sciences) and then a 1:1 mixture of Araldite (Electron Microscopy Sciences) and propylene oxide $(1 \mathrm{~h})$. Sections were infiltrated with Araldite overnight, followed by flat embedding in Araldite in aclar (Ted Pella). Aclarembedded tissue was cured for $48 \mathrm{~h}$ at $60^{\circ} \mathrm{C}$. Small pieces of tissue $500-750 \mu \mathrm{m}$ wide were cut from each section, reembedded in Araldite blocks, and cured for $48 \mathrm{~h}$ at $60^{\circ} \mathrm{C}$.

Serial sectioning and data analysis. To reconstruct postsynaptic sites, Araldite blocks containing embedded tissue from MDmc were sectioned at $50 \mathrm{~nm}$ using an ultramicrotome (Ultracut UCT; Leica Microsystems) and collected on pioloform-coated copper slot grids, to form series of $\sim 75-150$ sections. Using $80 \mathrm{kV}$ transmission EM at 16,000X$26,000 \times(100 \mathrm{CX}$; Jeol), a few sections were sampled to identify $\sim 10-30$ labeled boutons in each series. Each bouton was photographed in serial sections through at least 20 sections. Labeled boutons were analyzed using Reconstruct to trace bouton profiles and their postsynaptic sites (Fiala, 2005). Synapses, axon terminals, and postsynaptic sites were identified using classical criteria (Peters et al., 1991). A two-tailed $z$-test for proportions was used to determine whether amygdalar axons targeted $\mathrm{CB}$ and PV neurons equally or if they innervated one type preferentially.

\section{Results}

\section{Amygdalar axons target labeled neurons in MDmc that project to $\mathrm{POFC}$}

The amygdala projects to $\mathrm{MDmc}$, which projects robustly to pOFC, but it is not known whether this pathway is sequential because other subcortical structures also project to MD and MD also projects to areas in addition to pOFC (Russchen et al., 1987; Erickson et al., 2004; Rovó et al., 2012). To address this issue, we injected retrograde tracers in pOFC and anterograde tracers in the amygdala simultaneously (Fig. 1A). We first investigated whether the projection systems overlap in MDmc. We found that amygdalar axons in MDmc terminated in dense patches, many of which overlapped with retrogradely labeled pOFC projection neurons (Fig. $2 A-C$ ) even though the injection sites were small within the respective structures (Fig. $1 F, L$ ). Using laser scanning confocal microscopy to analyze areas of overlap, we found labeled amygdalar axons forming close appositions with labeled neurons in MDmc that project to pOFC (Fig. 2D,E). Only the cell body and proximal dendrites of retrogradely labeled neurons in MDmc that project to pOFC were labeled by this method, so only proximal targets of amygdalar axons could be identified.
To further characterize the overlap between amygdalar terminations and neurons in MDmc that project to pOFC, we calculated the proportion of pOFC projection neurons that were within $100 \mu \mathrm{m}$ of a labeled amygdalar terminal. We found that $44 \%$ of labeled neurons in MDmc that project to pOFC met this criterion ( $n=386$ of 877 pOFC projection neurons in MD), suggesting that these pathways overlap substantially in MD (Fig. $2 A-C)$. These findings provide evidence of a sequential pathway from the amygdala to pOFC through $\mathrm{MD}$, which includes strong proximal inputs on relay neurons in MD that project to pOFC. Therefore, in addition to sending signals to pOFC directly, the amygdala sends signals indirectly to pOFC through MDmc.

\section{Two thalamic pathways from $\mathrm{CB}$ and $\mathrm{PV}$ neurons project to pOFC}

We then investigated the types and prevalence of thalamic neurons from MDmc that project to pOFC. Unlike the cortex, in the primate thalamus, $\mathrm{CB}$ and $\mathrm{PV}$ neurons form two classes of excitatory projection neurons. $\mathrm{CB}$ neurons project diffusely and mostly to the upper layers of cortex, whereas $\mathrm{PV}$ neurons project focally to the middle cortical layers (Jones and Hendry, 1989; for discussion see Jones, 1998). Injections of tracers covering all cortical layers in pOFC (Fig. $1 D-H$ ) labeled projection neurons retrogradely in the thalamic MD nucleus. We then labeled tissue through the thalamus for $\mathrm{CB}$ or $\mathrm{PV}$ to study the proportion of projection neurons that were double labeled (Fig. $3 A-C$ ). Among double-labeled neurons in $\mathrm{MD}$, in each case, there was a consistent trend of a greater proportion of CB-positive projection neurons among retrogradely labeled neurons (CB-positive: mean \pm SEM, $36.1 \pm 7.5 \%$; PV-positive: $13.8 \pm 3.0 \% ; n=3$ cases; twotailed $t$ test, $t_{(2)}=2.4, p=0.1 ;$ Fig. $3 C$ ). To investigate the identity of the remaining retrogradely labeled neurons, in one case, we 

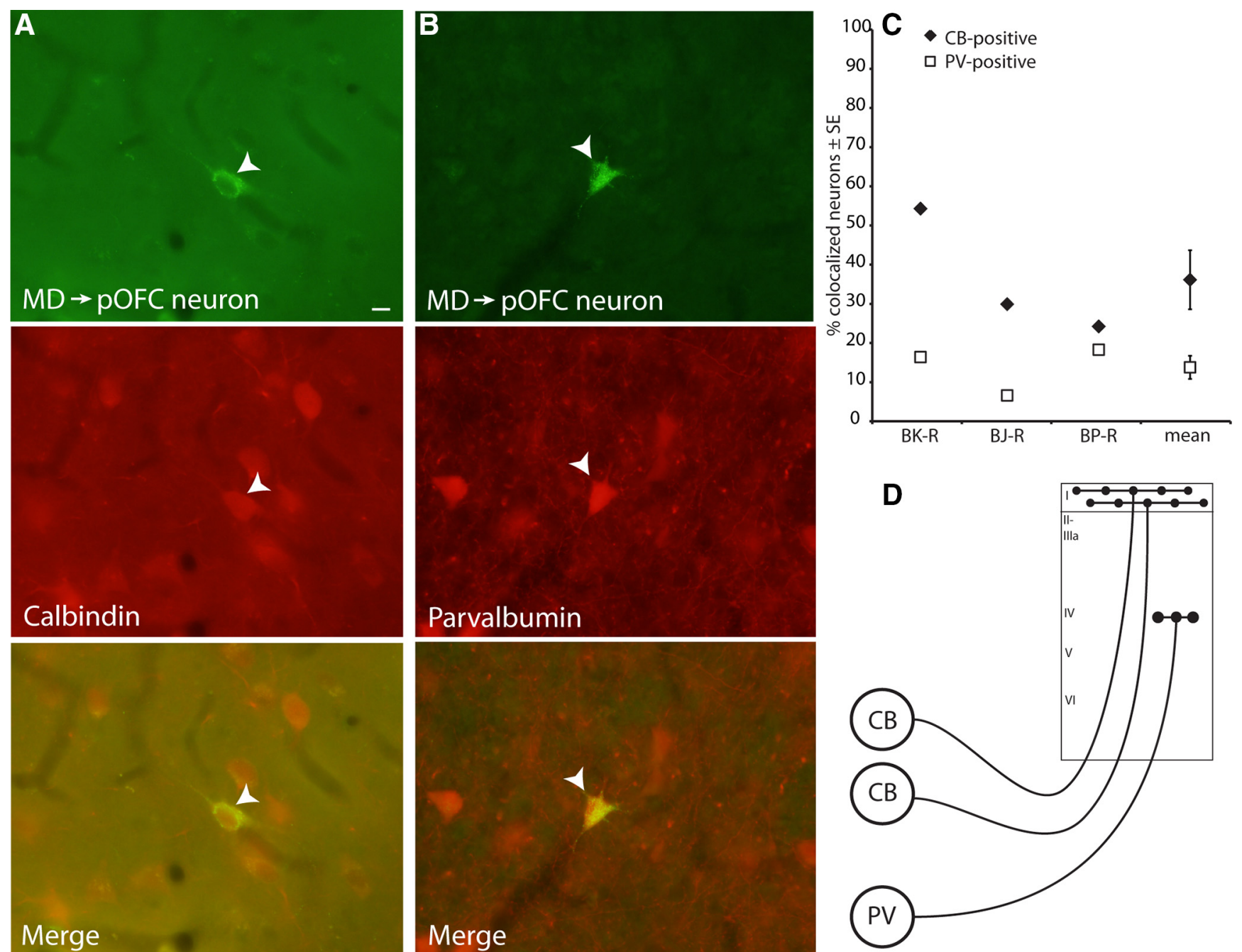

\section{Merge}

\section{Merge}

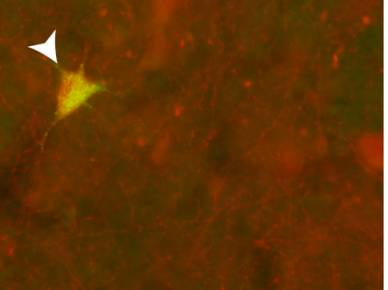

Figure 3. Neurons in MD projecting to pOFC colocalized with CB or PV. $A$, Thalamic MD neuron projecting to pOFC (top, white arrowhead) is also positive for $\mathrm{CB}$ (middle), as seen in the merged images (bottom). $\boldsymbol{B}$, Thalamic MD neuron projecting to POFC (top, white arrowhead) is also positive for PV (middle), as seen in the merged images (bottom). Scale bar in $\boldsymbol{A}$ is $10 \mu \mathrm{m}$ and applies to $\boldsymbol{A}$ and $\boldsymbol{B}$. C, Thalamic neurons projecting to POFC more frequently colocalized with CB than PV, in all cases analyzed. Vertical lines indicate SEM. D, Schematic depicting the termination pattern of calbindin thalamocortical neurons, which target robustly the upper cortical layers, and parvalbumin thalamocortical neurons, which terminate focally in the middle cortical layers.

also double labeled for CR and found that $3.1 \%$ of retrogradely labeled neurons were positive for $\mathrm{CR}$. The remaining projection neurons in MD that were directed to pOFC were unlabeled; this may represent lightly labeled CB or PV neurons that did not reach threshold to determine possible colocalization. Alternatively, there may be thalamic projection neurons that do not colocalize with any of the known calcium-binding proteins (CB, PV, or $\mathrm{CR}$ ), a question that remains to be addressed in future studies.

In one additional case, the tracer was confined to layers 5 and 6 of pOFC and the white matter below (case BC-R; Fig. 1C). In this case, $38 \%$ of double-labeled neurons in MD were PVpositive and comparatively fewer (29\%) were CB-positive. This evidence suggests that PV neurons in MD target the middle and deep cortical layers preferentially, whereas CB-positive neurons project diffusely to the upper and to the deep layers of pOFC. Previous studies have shown that MD terminals are densest in the middle layers of pOFC and are comparatively less dense in the upper cortical layers (Giguere and Goldman-Rakic, 1988). However, we found a tendency for a higher proportion of CB compared with $\mathrm{PV}$ projection neurons in MD directed to pOFC. One possible explanation for this finding may be related to the focal projection of $\mathrm{PV}$ neurons in the middle layers contrasted by the widespread projection of $\mathrm{CB}$ neurons to the upper layers, as well as the additional projection to the deep layers. Therefore, cortical injections label more CB neurons in MD, as depicted schematically in Figure $3 D$.

\section{Amygdalar pathways form synapses with excitatory CB and $\mathrm{PV}$ projection neurons in MDmc}

We next investigated whether the amygdala innervates both $\mathrm{CB}$ and PV neurons in MD or preferentially one type over another. To address this issue, we labeled the amygdalar pathway to MDmc with anterograde tracers and identified its postsynaptic targets in the EM by morphology and for the presence of label for $\mathrm{CB}$ or PV in thalamic projection neurons (Fig. 4). All amygdalar boutons formed excitatory synapses in MDmc. Further, this analysis revealed that the majority of amygdalar axons formed at least one synapse with an excitatory dendrite in $\operatorname{MDmc}(89 \%, n=34$ of 38 boutons; Fig. 4E). Amygdalar axons did not target $\mathrm{CB}$ and $\mathrm{PV}$ neurons equally: $\mathrm{CB}$ dendrites in $\mathrm{MDmc}$ were innervated significantly more frequently than PV dendrites ( $\mathrm{CB}=68 \%, n=$ 21 of 31 labeled dendrites; the remainder innervated PV dendrites; two-tailed $z$-test, $z=2.0, p=0.046$; Fig. $4 A-D$ ). This ratio closely matches the relative proportion of CB- and PV-positive 
neurons in MDmc that project to pOFC. This finding suggests that amygdalar input to MDmc preferentially and most robustly influences the upper cortical layers and, to a lesser extent, the deep layers through synapses on $\mathrm{CB}$ thalamic projection neurons. However, about one-third of amygdalar boutons innervated PV neurons in $\mathrm{MDmc}$, which project focally to the middle layers of pOFC.

\section{Amygdalar pathways to $\mathrm{pOFC}$ and to MD are distinct}

We next investigated whether pathways from the amygdala to the thalamic MDmc and to pOFC overlap or are distinct. To address this issue, we first investigated whether neurons in the amygdala project to both MDmc and to pOFC after comparatively large injections of distinct tracers in MD and pOFC (case BS-L; Fig. $1 G, M)$. We mapped retrogradely labeled neurons in the amygdala $(n=882 ; 422$ projected to $\mathrm{MDmc} ; 460$ projected to pOFC). Neurons directed to MDmc or pOFC were intermingled in the basolateral, basomedial (also known as accessory basal), cortical, and lateral amygdalar nuclei (Fig. $5 D-F)$, but none projected to both structures (Fig. 5A-C). This evidence suggests that pathways to MDmc and to pOFC arise from separate populations in the amygdala.

We then tested whether the two populations of neurons in the amygdala differ in size by analysis of surface area. Labeled neurons in the amygdala projecting to MD were significantly larger than those projecting to pOFC $(n=882$ neurons; MD-projecting neurons: mean $\pm \mathrm{SEM}, 287.7 \mu \mathrm{m}^{2} \pm 7.2$; pOFC-projecting neurons: $208.2 \mu \mathrm{m}^{2} \pm 3.4$; two-tailed $t$ test, $t_{(2)}=2.0, p<$ 0.001 ; Fig. $5 G$ ).

The morphological differences between amygdalar neurons that project to MDmc and those that project to pOFC suggest that these pathways may also be distinct by the specificity of their glutamatergic terminations. Although both of these pathways are excitatory and glutamatergic, we investigated whether they could be further distinguished by expression of VGLUT1 or VGLUT2, which is unknown for these pathways. To address this issue, we used immunofluorescence to investigate colocalization between labeled amygdalar axon terminals and VGLUT1 or VGLUT2 in pOFC and MDmc ( $n=1626$ boutons from two cases; Fig. 6). We found that, in the upper layers of pOFC, labeled amygdalar axons colocalized more frequently with VGLUT1 (33\% VGLUT1, $n=161$ of 485 boutons from 2 cases; 3\% VGLUT2, $n=16$ of 611 boutons from 2 cases; $\chi_{(1, n=1096)}^{2}=186.7, p<0.0001$; Fig. $\left.6 A, B, E, F\right)$. The remainder boutons were unlabeled and may represent boutons that were lightly labeled by VGLUT1 or VGLUT2 and did not meet detection for colocalization. In contrast, in MDmc, labeled amygdalar axons colocalized frequently with VGLUT2 (70\% VGLUT2, $n=179$ of 257 boutons from 2 cases; $0.4 \%$ VGLUT1, $n=1$ of 273 boutons from 2 cases; $\chi_{(1, n=530)}^{2}=283.3, p<$ 0.0001; the remainder were unlabeled; Fig. $6 C-F$ ). This finding reveals that the dual amygdalar pathways that terminate in pOFC and MDmc differ by their glutamate transporter. In conjunction with the morphological differences between the two pathways, these findings support the idea that amygdalar projections to cortex and MDmc arise from distinct neurons in the amygdala, as has been proposed in rats (McDonald, 1987).

\section{Discussion}

Our findings show that a pathway from the amygdala targets thalamic neurons in MDmc that project to pOFC, providing evidence for an indirect route through which signals from the amygdala reach pOFC (Fig. 7). The tripartite system that links the amygdala, MDmc, and pOFC showed novel specializations, as discussed below.

\section{Direct and indirect routes connect the amygdala with pOFC}

The primate amygdala projects to $\mathrm{MDmc}$, which is reciprocally connected with pOFC (Giguere and Goldman-Rakic, 1988; Dermon and Barbas, 1994). However, because other inputs also converge on $\mathrm{MDmc}$ and neurons in $\mathrm{MD}$ project to other areas (Russchen et al., 1987; Erickson et al., 2004; Rovó et al., 2012), it was uncertain whether an indirect pathway connects the amygdala with pOFC through MD. We provide direct evidence about the existence of a sequential pathway between the basal and cortical nuclei of the amygdala to pOFC through MD. This indirect route may allow the amygdala to activate thalamic afferents to $\mathrm{pOFC}$, along with a direct amygdalar pathway to pOFC.

The two pathways from MDmc and the amygdala differ in their preferred laminar distribution and interactions with distinct classes of inhibitory neurons in pOFC (Timbie and Barbas, 2014). Amygdalar pathways terminate most densely in the upper layers of pOFC (Porrino et al., 1981; Ghashghaei et al., 2007), form synapses largely with excitatory neurons, and their terminals are even larger than thalamic terminals in the middle cortical layers (Timbie and Barbas, 2014), which are considered to be 

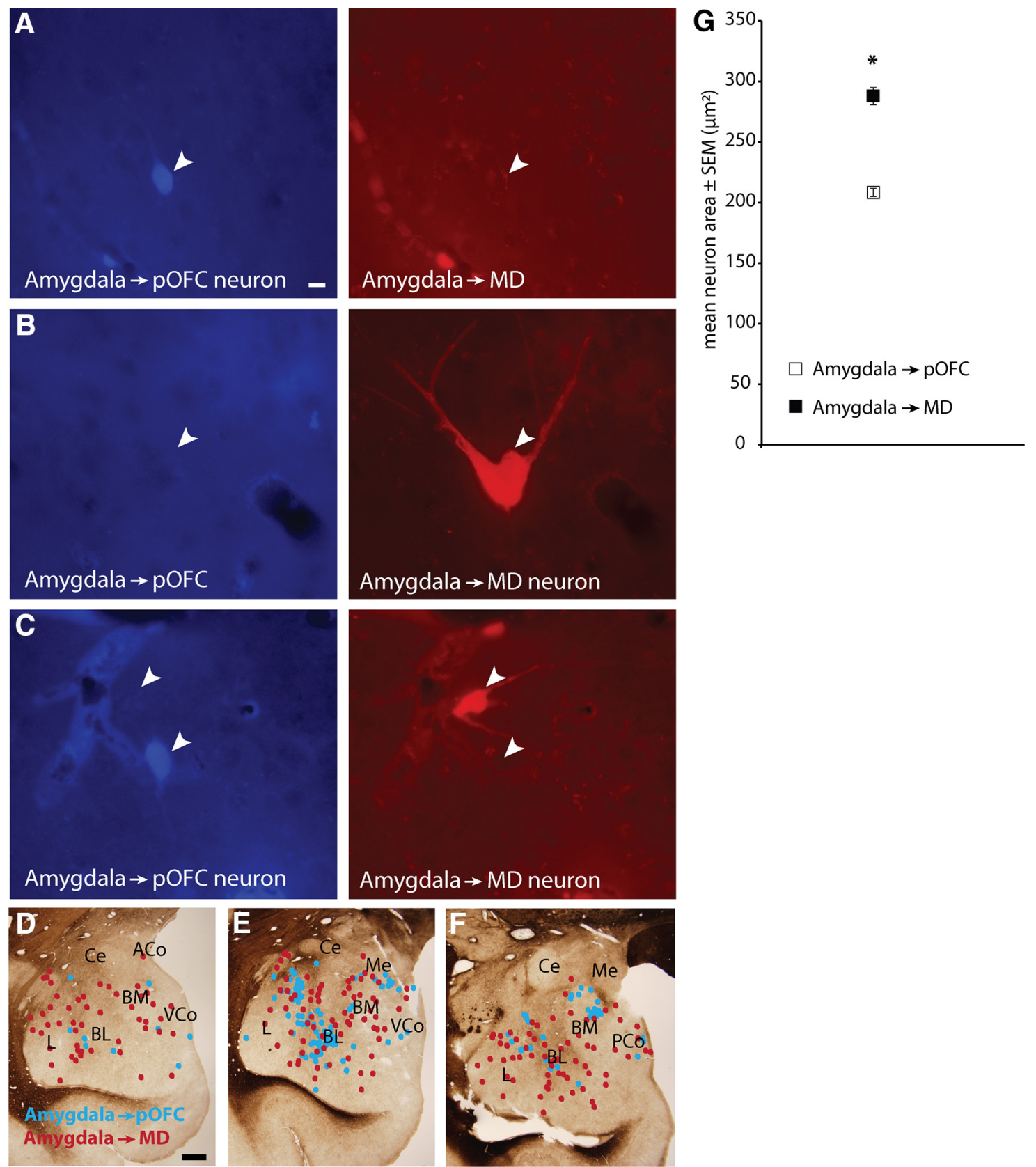

Figure 5. Amygdalar neurons that project to MD and to pOFC are distinct. $A$, Amygdalar neuron is labeled with tracer injected in pOFC (left, blue, white arrowhead), but is not labeled with tracer from MD (right). Scale bar in $A$ is $10 \mu \mathrm{m}$ and applies to $A-C . B$, Amygdalar neuron is labeled with tracer injected in MD (right, red, white arrowhead), but is not labeled with tracer from p0FC (left). C, Neurons in the same field show that different neurons in the amygdala were labeled retrogradely with tracer from pOFC (left, bottom arrowhead) or from MD (right, top arrowhead); neither neuron is double-labeled. $\boldsymbol{D}-\boldsymbol{F}$, Representative coronal sections from fresh tissue through the amygdala (anterior $\rightarrow$ posterior) show spatial overlap of projection neurons directed to MD (red dots) or pOFC (blue dots). Scale bar in $\boldsymbol{D}$ is $1 \mathrm{~mm}$ and applies to $\boldsymbol{D}-\boldsymbol{F}$. G, Mean area of labeled amygdalar projection neurons directed to MD was significantly larger than for projection neurons directed to pOFC. Vertical lines indicate SEM.

“drivers" (Jones, 1998; Sherman and Guillery, 1998). The amygdala is thus poised to have a significant impact on all layers of pOFC, in the upper layers through large terminals via both a direct pathway and an indirect pathway by activating MD that projects to the middle and upper layers.

Our findings showed that both the $\mathrm{CB}$ and PV systems from MDmc project to pOFC, such as the relay thalamic nuclei to sensory areas, which mark excitatory projection neurons in two parallel thalamocortical pathways: axons from CB neurons terminate mostly in the upper cortical layers, whereas axons from PV neurons terminate in the middle layers (Jones and Hendry,
1989). Unlike the pattern for sensory areas, we found that there was a tendency for a higher prevalence of $\mathrm{CB}$ neurons in MDmc that project to $\mathrm{POFC}$, suggesting a bias of innervation of the upper and deep layers. The CB bias may reflect differences in the pattern of innervation by the two thalamic systems. Therefore, whereas the axon of each thalamic PV neuron terminates in a dense patch focally in the middle cortical layers, each thalamic CB neuron terminates in a widespread arbor covering the upper cortical layers. The broad terminations of each CB neuron thus overlap and extend beyond the narrow column of axon terminations in the middle layers from each PV neuron, as shown schemati- 

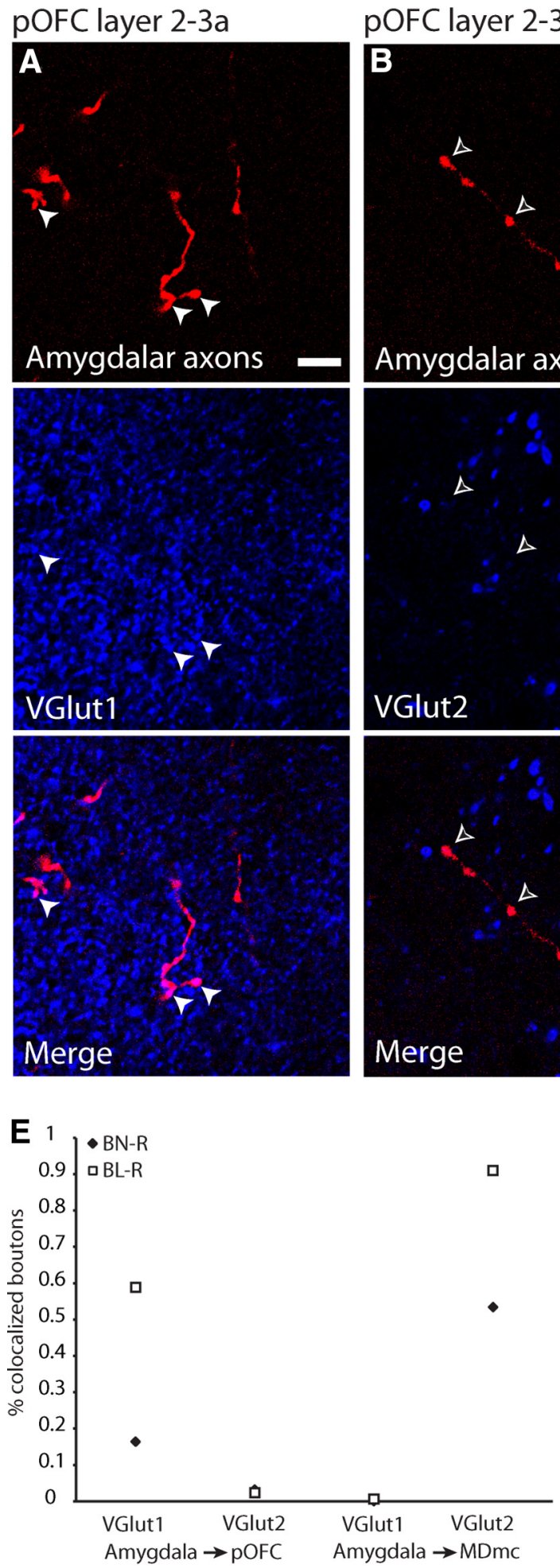

pOFC layer 2-3a

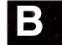

Amygdalar axons
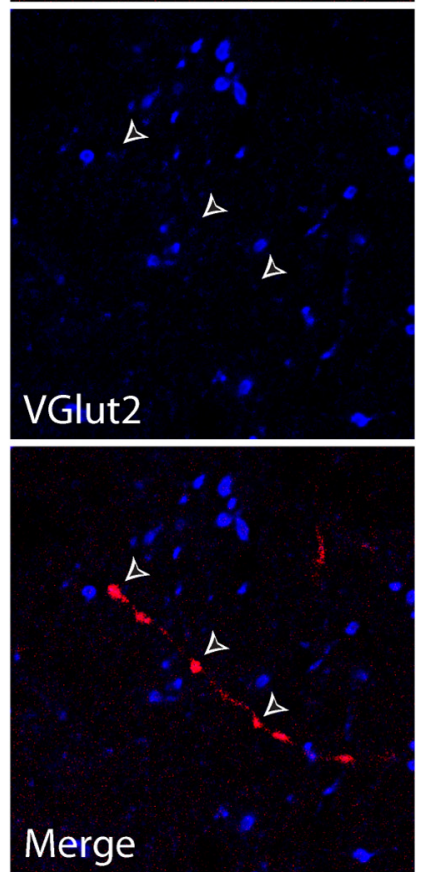

Thalamus MDmc
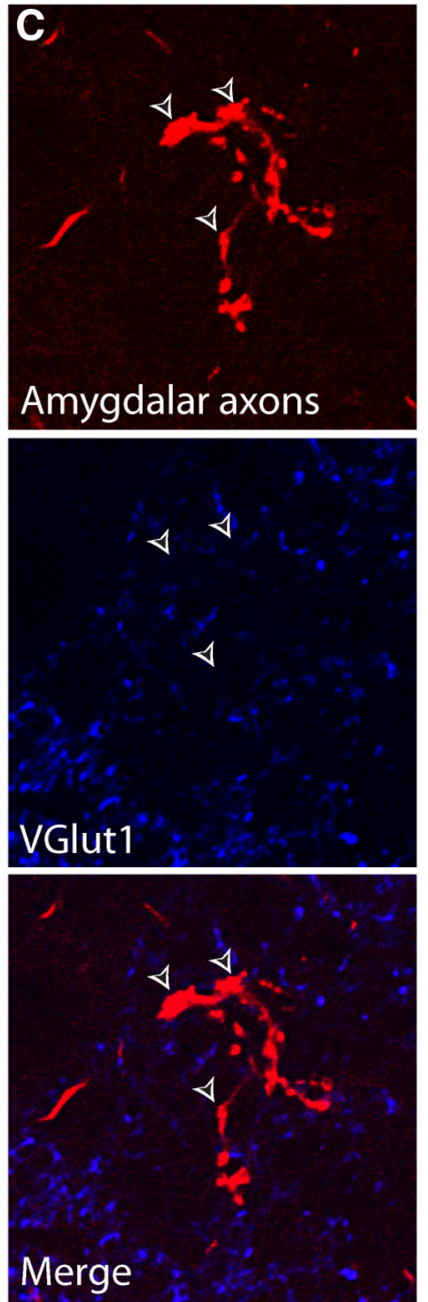

Thalamus MDmc
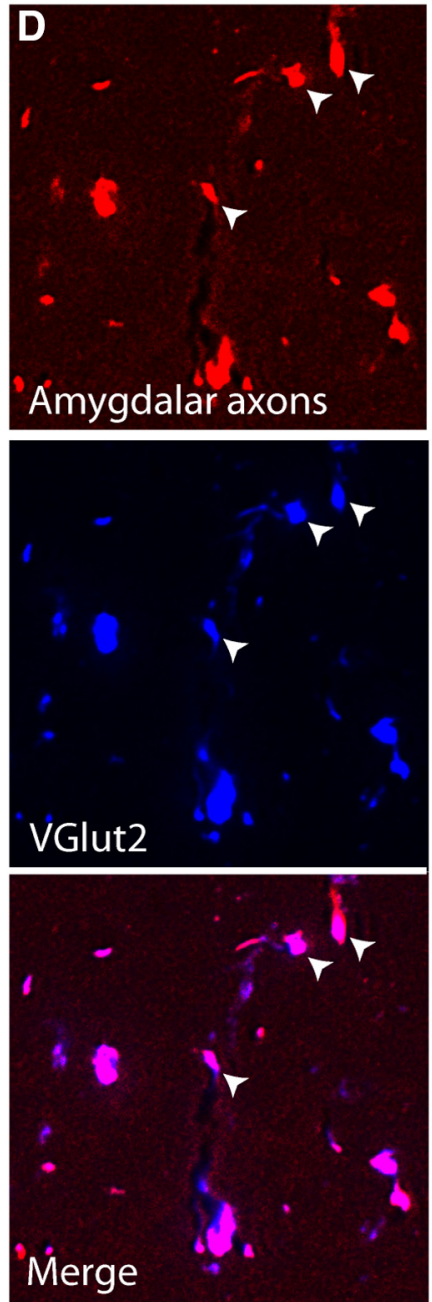

$\mathbf{F}$

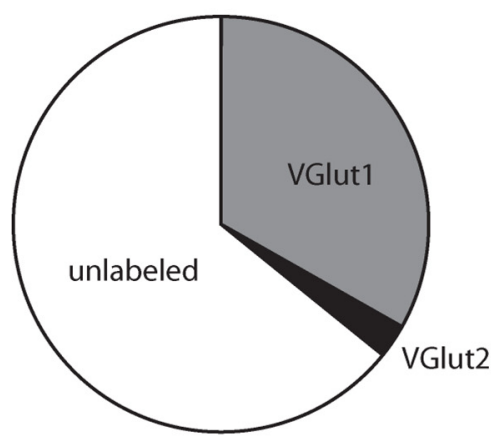

Amygdala $\rightarrow$ pOFC

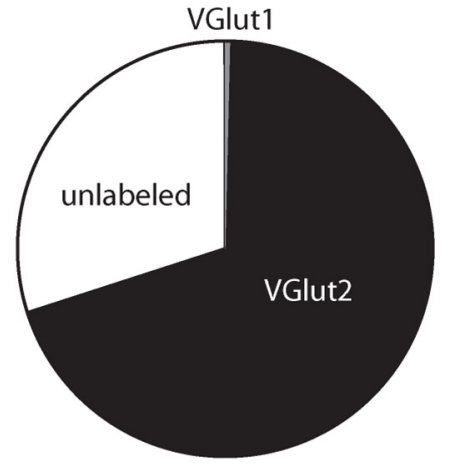

Amygdala $\rightarrow$ MDmc

Figure 6. Amygdalar axons in MDmc and pOFC express different glutamate transporters. $A, B$, Amygdalar axons (top, white arrowheads) in the upper layers of pOFC colocalized with VGLUT1 (middle), but not with VGLUT2 (middle, silhouette arrowheads), as seen in the merged images (bottom). Scale bar in $A$ is $10 \mu \mathrm{m}$ and applies to all panels. C, D, Amygdalar axons in MDmc (top, white arrowheads) colocalized with VGLUT2 (middle, white arrowheads), but not with VGLUT1 (middle, silhouette arrowheads), as seen in the merged images (bottom). $\boldsymbol{E}$, Scatterplot depicts the proportion of amygdalar boutons in each case which colocalized with VGLUT1 or VGLUT2 in MDmc or pOFC. F, Overall proportion of amygdalar boutons that colocalized with VGLUT1 or VGLUT2 in MDmc or pOFC.

cally in Figure 3D. Further, we provide evidence for a novel calretinin-positive pathway emanating from a small number of neurons in MDmc directed to pOFC.

The predominance of $\mathrm{CB}$-positive projection neurons to pOFC may be a specialized projection to limbic cortices, which complete their development earlier than the six-layered eulaminate cortices (Rakic, 2002), as had been hypothesized on the basis of their laminar structure (Dombrowski et al., 2001; Barbas, 2015). This timing coincides more closely with the earlier development of CB neurons than PV neurons in the human thalamus 


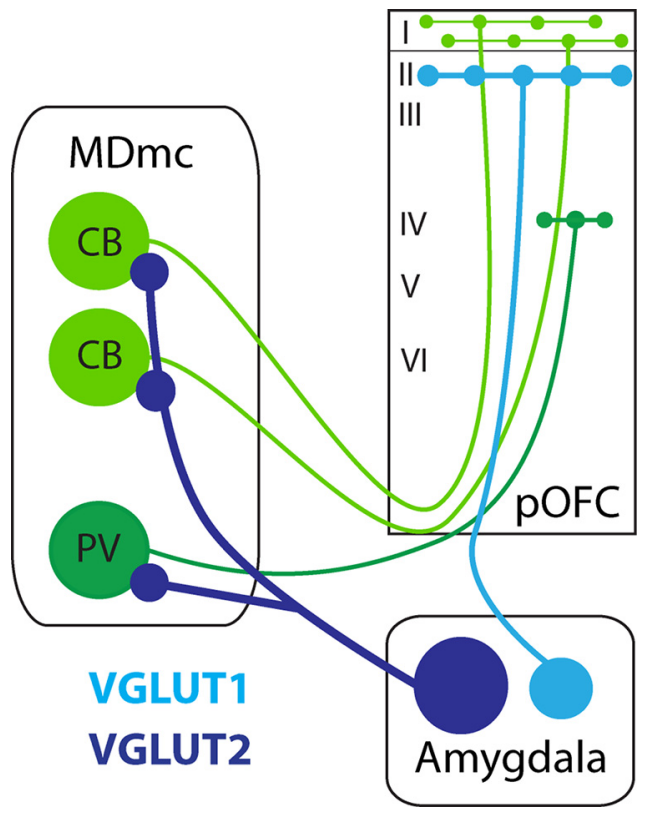

Figure 7. Amygdalar signals reach pOFC directly and indirectly through the thalamus. Bottom, Neurons from the amygdala that project to the thalamus are large and express VGLUT2 (dark blue), whereas amygdalar neurons that project to pOFC are comparatively smaller and express VGLUT1 (light blue). Left, Excitatory CB neurons (light green) and excitatory PV neurons (dark green) in MDmc project to $\mathrm{pOFC}$ at a ratio of 2:1. Direct amygdalar projections target most densely the upper layers, whereas indirect projections through the thalamus may target upper and middle layers through CB and PV thalamic neurons to enhance cortical signaling of affective content.

(Kultas-Ilinsky et al., 2004). In addition, the lack of a well developed layer 4 in limbic cortices could shift thalamic input to other layers.

\section{Direct and indirect routes that connect the amygdala with pOFC are distinct}

The direct and indirect amygdalar pathways to pOFC differ by morphology. Neurons in the amygdala that innervated MD were larger than those that projected to pOFC. Further, the two amygdalar pathways differed in their expression of vesicular glutamate transporters: the amygdalar terminals in MDmc expressed predominantly VGLUT2, whereas amygdalar terminals to cortex were mostly positive for VGLUT1. This evidence suggests that the presence of a specific glutamate transporter in pathways that originate from a given region depends on the destination and specific type of innervation by each pathway.

The functional significance of this finding is based on the fundamental role of glutamate transporters to fill vesicles with glutamate and maintain the readily releasable pool of vesicles at axon terminals of glutamatergic neurons (Fremeau et al., 2001; Varoqui et al., 2002; Fremeau et al., 2004; Weston et al., 2011). Corticocortical and corticothalamic axons express VGLUT1, whereas thalamocortical axons express primarily VGLUT2 in primates (Hackett et al., 2011). Compared with VGLUT1 terminals, VGLUT2 thalamocortical terminals have a higher probability of vesicular release (Weston et al., 2011). This suggests that VGLUT2 terminals from the amygdala are highly efficient and likely elicit action potentials in their postsynaptic targets in $\mathrm{MD}$, ensuring transmission of signals. In the primate visual system, pathways with VGLUT2 terminals have been likened to feedforward "driver" connections and those with VGLUT1 with modulator "feedback" pathways (Balaram et al., 2013). Our finding of dissociation of amygdalar pathways to pOFC and to MDmc by their vesicular neurochemistry reinforces the suggestion that the two pathways are distinct in both rats and monkeys (McDonald, 1987; Miyashita et al., 2007).

The morphological and chemical differences suggest that neurons in the amygdala that project to the thalamus or cortex may also differ physiologically. Two types of excitatory projection neurons have been described in the basolateral amygdala of cats and rats: bursting and regular spiking (Rainnie et al., 1993; Paré et al., 1995). Bursting neurons are larger and have a lower input resistance, so they may be less excitable (Rainnie et al., 1993). There is evidence for the presence of two such groups in the hippocampal subiculum as well, in which bursting neurons project to presubiculum, whereas regular spiking neurons project to the entorhinal cortex (for review, see O'Mara et al., 2001). By analogy, large neurons in the amygdala that project to MD may be bursting neurons that respond to sensory stimuli with affective content (Gonzalez Andino and Grave de Peralta Menendez, 2012). The possibility that bursting amygdalar neurons project to MDmc provides a physiologic mechanism for salient stimuli to activate thalamic neurons for transmission to pOFC.

\section{Role of the high-order MDmc thalamic nucleus in emotion}

The central node in the tripartite circuit, the thalamic MDmc nucleus, has an important role in processing affective content. Neurons in MDmc in rats fire differentially in response to stimuli associated with reward (Oyoshi et al., 1996; Li et al., 2004), similar to neurons in the primate amygdala and orbitofrontal cortex (Belova et al., 2008; Morrison and Salzman, 2011). However, thalamic neurons differ from cortical and amygdalar neurons in their physiological properties. Unlike the amygdala, thalamic projection neurons may switch between firing in bursting or tonic modes, which elicit different network effects (Steriade et al., 1997; Jones, 2009).

The nature of signals sent through the sequential pathway from the amygdala to MDmc and pOFC is not known, but other thalamic pathways may offer clues. A sequential pathway from the primate superior colliculus innervates another part of $\mathrm{MD}$, the lateral sector that projects to the frontal eye fields (FEF; Sommer and Wurtz, 2004). In this sequential pathway, it has been suggested that MD acts as a high-pass filter to transmit highfrequency signals from the superior colliculus to the FEF. Signals sent along this pathway are thought to represent corollary discharges related to planned saccades (Sommer and Wurtz, 2004). By analogy, amygdalar neurons may send corollary signals through MDmc to transmit affective content and recruit relevant cortical areas and ensure that signals arriving via the direct amygdalo-pOFC pathway are received. A similar role has been proposed for the pulvinar thalamic nucleus: amygdalar connections with pulvinar projection neurons may activate visual cortices to coordinate cortical processing of visual stimuli with emotional relevance (Pessoa and Adolphs, 2010).

Lesions of MD impair conditioned fear learning in rats ( $\mathrm{Li}$ et al., 2004) and, in primates, they impair memory and reinforcement devaluation, a process that requires updating the value of rewards (Markowitsch, 1982; Zola-Morgan and Squire, 1985; Izquierdo and Murray, 2010; for review, see Mitchell and Chakraborty, 2013). Deficits in reward processing may be related to loss of the sequential amygdalar pathway to MDmc and pOFC in lesioned animals. In this context, mice with selective deletion of VGLUT2 show changes in social behavior, including increased risk taking and decreased avoidance behavior (WallénMackenzie et al., 2009), which may be attributed to 
disconnection in the pathway from the amygdala to pOFC through MDmc. Conversely, increasing activity in MD produces the opposite behavioral changes, including anxiety-like symptoms, supporting a role of the sequential pathway in emotional behavior (Rotge et al., 2012). Our findings suggest that the strong and efficient excitatory pathway from the amygdala to MD activates cortically projecting neurons, including those that project widely to the upper layers of cortex, and may be necessary for recruiting cortical areas during tasks with affective import. Further, the finding of direct and indirect amygdalar pathways suggests enhanced access of stimuli with affective significance to all layers of pOFC.

\section{References}

Aggleton JP, Mishkin M (1984) Projections of the amygdala to the thalamus in the cynomolgus monkey. J Comp Neurol 222:56-68. CrossRef Medline

Balaram P, Hackett TA, Kaas JH (2013) Differential expression of vesicular glutamate transporters 1 and 2 may identify distinct modes of glutamatergic transmission in the macaque visual system. J Chem Neuroanat 50-51:21-38. CrossRef Medline

Barbas H (2015) General cortical and special prefrontal connections: principles from structure to function. Annu Rev Neurosci 38:269-289. CrossRef Medline

Baxter MG, Murray EA (2002) The amygdala and reward. Nat Rev Neurosci 3:563-573. CrossRef Medline

Belova MA, Paton JJ, Salzman CD (2008) Moment-to-moment tracking of state value in the amygdala. J Neurosci 28:10023-10030. CrossRef Medline

Davis M, Whalen PJ (2001) The amygdala: vigilance and emotion. Mol Psychiatry 6:13-34. CrossRef Medline

Dermon CR, Barbas H (1994) Contralateral thalamic projections predominantly reach transitional cortices in the rhesus monkey. J Comp Neurol 344:508-531. CrossRef Medline

Dombrowski SM, Hilgetag CC, Barbas H (2001) Quantitative architecture distinguishes prefrontal cortical systems in the rhesus monkey. Cereb Cortex 11:975-988. CrossRef Medline

Erickson SL, Melchitzky DS, Lewis DA (2004) Subcortical afferents to the lateral mediodorsal thalamus in cynomolgus monkeys. Neuroscience 129: 675-690. CrossRef Medline

Fiala JC (2005) Reconstruct: a free editor for serial section microscopy. J Microsc 218:52-61. CrossRef Medline

Fremeau RT Jr, Troyer MD, Pahner I, Nygaard GO, Tran CH, Reimer RJ, Bellocchio EE, Fortin D, Storm-Mathisen J, Edwards RH (2001) The expression of vesicular glutamate transporters defines two classes of excitatory synapes. Neuron 31:247-260. CrossRef Medline

Fremeau RT Jr, Voglmaier S, Seal RP, Edwards RH (2004) VGLUTs define subsets of excitatory neurons and suggest novel roles for glutamate. Trends Neurosci 27:98-103. CrossRef Medline

Ghashghaei HT, Hilgetag CC, Barbas H (2007) Sequence of information processing for emotions based on the anatomic dialogue between prefrontal cortex and amygdala. Neuroimage 34:905-923. CrossRef Medline

Giguere M, Goldman-Rakic PS (1988) Mediodorsal nucleus: Areal, laminar, and tangential distribution of afferents and efferents in the frontal lobe of rhesus monkeys. J Comp Neurol 277:195-213. CrossRef Medline

Gonzalez Andino SL, Grave de Peralta Menendez R (2012) Coding of saliency by ensemble bursting in the amygdala of primates. Front Behav Neurosci 6:38. Medline

Hackett TA, Takahata T, Balaram P (2011) VGLUT1 and VGLUT2 mRNA expression in the primate auditory pathway. Hear Res 274:129-141. CrossRef Medline

Izquierdo A, Murray EA (2010) Functional interaction of medial mediodorsal thalamic nucleus but not nucleus accumbens with amygdala and orbital prefrontal cortex is essential for adaptive response selection after reinforcer devaluation. J Neurosci 30:661-669. CrossRef Medline

Jensen FE, Harris KM (1989) Preservation of neuronal ultrastructure in hippocampal slices using rapid microwave-enhanced fixation. J Neurosci Methods 29:217-230. Medline

Jiao Y, Sun Z, Lee T, Fusco FR, Kimble TD, Meade CA, Cuthbertson S, Reiner A (1999) A simple and sensitive antigen retrieval method for freefloating and slide-mounted tissue sections. J Neurosci Methods 93:149162. CrossRef Medline
Jones EG (1998) Viewpoint: the core and matrix of thalamic organization. Neuroscience 85:331-345. CrossRef Medline

Jones EG (2009) Synchrony in the interconnected circuitry of the thalamus and cerebral cortex. Am N Y Acad Sci 1157:10-23. CrossRef Medline

Jones EG, Hendry SH (1989) Differential calcium binding protein immunoreactivity distinguishes classes of relay neurons in monkey thalamic nuclei. Eur J Neurosci 1:222-246. CrossRef Medline

Kultas-Ilinsky K, Fallet C, Verney C (2004) Development of the human motor-related thalamic nuclei during the first half of gestation, with special emphasis on GABAergic circuits. J Comp Neurol 476:267-289. CrossRef Medline

Li XB, Inoue T, Nakagawa S, Koyama T (2004) Effect of mediodorsal thalamic nucleus lesion on contextual fear conditioning in rats. Brain Res 1008:261-272. CrossRef Medline

Markowitsch HJ (1982) Thalamic mediodorsal nucleus and memory: A critical evaluation of studies in animals and man. Neurosci Biobehav Rev 6:351-380. CrossRef Medline

McDonald AJ (1987) Organization of amygdaloid projections to the mediodorsal thalamus and prefrontal cortex: a fluorescence retrograde transport study in the rat. J Comp Neurol 262:46-58. CrossRef Medline

McFarland NR, Haber SN (2002) Thalamic relay nuclei of the basal ganglia form both reciprocal and nonreciprocal cortical connections, linking multiple frontal cortical areas. J Neurosci 22:8117-8132. Medline

Mitchell AS, Chakraborty S (2013) What does the mediodorsal thalamus do? Front Syst Neurosci 7:37. Medline

Miyashita T, Ichinohe N, Rockland KS (2007) Differential modes of termination of amygdalothalamic and amygdalocortical projections in the monkey. J Comp Neurol 502:309-324. CrossRef Medline

Morrison SE, Salzman CD (2011) Representations of appetitive and aversive information in the primate orbitofrontal cortex. Ann N Y Acad Sci 1239:59-70. CrossRef Medline

National Research Council (2011) Guide for the care and use of laboratory animals, Ed 8. Washington, DC: National Academies.

Olszewski J (1952) The thalamus of the Macaca mulatta: an atlas for use with the stereotaxic instrument. Basel: Karger.

O’Mara SM, Commins S, Anderson M, Gigg J (2001) The subiculum: a review of form, physiology and function. Prog Neurobiol 64:129-155. CrossRef Medline

Oyoshi T, Nishijo H, Asakura T, Takamura Y, Ono T (1996) Emotional and behavioral correlates of mediodorsal thalamic neurons during associative learning in rats. J Neurosci 16:5812-5829. Medline

Paré D, Pape HC, Dong J (1995) Bursting and oscillating neurons of the cat basolateral amygdaloid complex in vivo: electrophysiological properties and morphological features. J Neurophysiol 74:1179-1191. Medline

Pessoa L, Adolphs R (2010) Emotion processing and the amygdala: from a "low road" to "many roads" of evaluating biological significance. Nat Rev Neurosci 11:773-783. Medline

Peters A, Palay SL, Webster HD (1991) The fine structure of the nervous system: neurons and their supporting cells. New York: Oxford UP.

Porrino LJ, Crane AM, Goldman-Rakic PS (1981) Direct and indirect pathways from the amygdala to the frontal lobe in rhesus monkeys. J Comp Neurol 198:121-136. CrossRef Medline

Rainnie DG, Asprodini EK, Shinnick-Gallagher P (1993) Intracellular recordings from morphologically identified neurons of the basolateral amygdala. J Neurophysiol 69:1350-1362. Medline

Rakic P (2002) Neurogenesis in adult primate neocortex: an evaluation of the evidence. Nat Rev Neurosci 3:65-71. Medline

Rasband WS (1997-2014) ImageJ. Bethesda: National Institutes of Health.

Rosene DL, Roy NJ, Davis BJ (1986) A cryoprotection method that facilitates cutting frozen sections of whole monkey brains from histological and histochemical processing without freezing artifact. J Histochem Cytochem 34:1301-1315. CrossRef Medline

Rotge JY, Aouizerate B, Amestoy V, Lambrecq V, Langbour N, Nguyen TH, Dovero S, Cardoit L, Tignol J, Bioulac B, Burbaud P, Guehl D (2012) The associative and limbic thalamus in the pathophysiology of obsessivecompulsive disorder: an experimental study in the monkey. Transl Psychiatry 2:e161. CrossRef Medline

Rovó Z, Ulbert I, Acsady L (2012) Drivers of the primate thalamus. J Neurosci 32:17894-17908. CrossRef Medline

Russchen FT, Amaral DG, Price JL (1987) The afferent input to the magnocellular division of the mediodorsal thalamic nucleus in the monkey, Macaca fascicularis. J Comp Neurol 256:175-210. CrossRef Medline 
Salzman CD, Fusi S (2010) Emotion, cognition, and mental state representation in amygdala and prefrontal cortex. Ann Rev Neurosci 33:173-202. CrossRef Medline

Santos MS, Li H, Voglmaier SM (2009) Synaptic vesicle protein trafficking at the glutamate synapse. Neuroscience 158:189-203. CrossRef Medline

Sherman SM, Guillery RW (1998) On the actions that one nerve cell can have on another: distinguishing "drivers" from "modulators." Proc Natl Acad Sci U S A 95:7121-7126. CrossRef Medline

Sommer MA, Wurtz RH (2004) What the brain stem tells the frontal cortex. I. Oculomotor signals sent from superior colliculus to frontal eye field via mediodorsal thalamus. J Neurophysiol 91:1381-1402. Medline

Steriade M, Jones EG, McCormick DA (1997) Thalamus: organisation and function. Oxford: Elsevier Science.

Timbie C, Barbas H (2014) Specialized pathways from the primate amygdala to posterior orbitofrontal cortex. J Neurosci 34:8106-8118. CrossRef Medline

Varoqui H, Schäfer MK, Zhu H, Weihe E, Erickson JD (2002) Identification of the differentiation-associated $\mathrm{Na}+/ \mathrm{PI}$ transporter as a novel vesicular glutamate transporter expressed in a distinct set of glutamatergic synapses. J Neurosci 22:142-155. Medline

Wallén-Mackenzie A, Nordenankar K, Fejgin K, Lagerström MC, Emilsson L,
Fredriksson R, Wass C, Andersson D, Egecioglu E, Andersson M, Strandberg J, Lindhe O, Schiöth HB, Chergui K, Hanse E, Langstrom B, Fredriksson A, Svensson L, Roman E, Kullander K (2009) Restricted cortical and amygdaloid removal of vesicular glutamate transporter 2 in preadolescent mice impacts dopaminergic activity and neuronal circuitry of higher brain function. J Neurosci 29:2238-2251. CrossRef Medline

West EA, DesJardin JT, Gale K, Malkova L (2011) Transient inactivation of orbitofrontal cortex blocks reinforcer devaluation in macaques. J Neurosci 31:15128-15135. CrossRef Medline

Weston MC, Nehring RB, Wojcik SM, Rosenmund C (2011) Interplay between VGLUT isoforms and endophilin A1 regulates neurotransmitter release and short-term plasticity. Neuron 69:1147-1159. CrossRef Medline

Wilson RC, Takahashi YK, Schoenbaum G, Niv Y (2014) Orbitofrontal cortex as a cognitive map of task space. Neuron 81:267-279. CrossRef Medline

Zola-Morgan S, Squire LR (1985) Amnesia in monkeys after lesions of the mediodorsal nucleus of the thalamus. Ann Neurol 17:558-564. CrossRef Medline 